\title{
Disability, sexuality and access to services
}

2003 is the European Year of the Disabled. It seems appropriate that these two editorials, which follow a half-day conference session on disability, sexuality and access to services held in the UK, should appear in 2003. That a third of a conference programme addressed this issue was a step forward in itself in raising awareness. The fact that three of the four presentations were by workers and trainers in the field who themselves have physical disabilities, namely spinal injury (see Editorial on page 125), visual impairment and profound deafness, gave these presentations more credibility and impact. The fourth speaker, highly regarded and experienced in the field of learning disability, spoke on consent and capacity to consent and raised the particular issues relating to learning disability (see Overview on page 148). These issues often cause anxiety and difficulty to clinicians endeavouring to provide a service to clients with special needs.

In October 2004, further parts of the Disability Discrimination Act $1995^{1}$ are to be introduced. This will mean that everyone is supposed to have access to whatever facility they want to use - be it hotels, pubs, restaurants, cinemas, churches, shops, transport and, of course, health facilities. This should ensure physical access, but access to a service is about more than having a ramp and a suitably modified toilet facility. Access is also about availability of equal information so that informed choices can be made, and feeling welcome, accepted and valued alongside other non-disabled service users. Whilst it can be helpful and sometimes necessary to provide specialist, dedicated services for people with a disability, e.g. home visiting, the majority want the ability to access general services with consideration for particular enabling measures. Incorrect assumptions are often made. The person most able to tell providers what they need is the service user. In general and individual consultations, asking if assistance is needed and in which way it needs to be given is often overlooked, forgetting that each individual is the expert as regards their own needs.

Apart from the abovementioned ramps and toilets, good signposting is essential. Asking the way can be difficult for some clients due to:

- anxiety about how they will be received or judged

- speech difficulties

- lack of vocabulary

- difficulty in hearing

- difficulty understanding replies

- inability to read.

Symbols can be valuable, for example, Makaton for people with learning disabilities. For clients with visual impairment, good lighting, use of colour (especially black on yellow) and bold lettering will help. Highlighting of steps and door handles facilitates movement. Braille labelling is an advantage, but the client needs to be aware that it is provided.

Before any client can consider accessing services they need to know that they exist and that their sexual needs are normal and acceptable. For some clients this will be a whole new concept. For people with congenital disabilities (which includes learning disability) sex education, if delivered at all, may not have been in an acceptable, understandable format or may have seemed irrelevant at the time. There may be no understanding of rights or needs. ${ }^{2}$ Unfortunately, even in 2003 , societal attitudes to disability still exist such that people with a disability are seen as asexual or non-sexual. This attitude makes it particularly difficult to access services, especially as many clients have poor self-esteem and self-confidence. Thankfully others are confident and rightly expect services to be accessible; these clients will access the relevant services but there is much to do to enable availability for all.

A particular difficulty can be the need to involve a third party, perhaps a parent or carer. How will they receive and act on the client's request? How many people wish to ask their parents to be involved in this private, sensitive area of their life?

Having reached a consultation, communication is a major issue. Some insight into anxieties about being understood, understanding, being able to make decisions or having decisions made for them is essential. These are common worries for people with learning disabilities. In this context an important issue is sexual health screening. Utilisation of resources to enable learningdisabled clients to be screened is valuable. ${ }^{3}$ Some lateral thinking in how to administer and manage drugs and appliances is necessary. An example of this was shown by a blind man being advised to use alprostadil pellets for treatment of his erectile failure. Although this had been discussed at a hospital appointment the patient was sent home with a leaflet, which was largely diagrammatic. This proved useless, as the scanner he used for letters could not cope with diagrams. The client thus had a method of treatment but no means of utilising it. Some forethought could have avoided this situation. Eventually help was sought and the patient quickly mastered the technique with a demonstrator applicator and a penile model.

Making services accessible and inclusive does not usually require major financial input. Indeed there may be none. Raised awareness and careful thought can make the attitudinal shift to make services inclusive and thus available and valuable to service users with disabilities.

\section{Statements on funding and competing interest}

Funding. None identified.

Competing interests. The author is a consultant for the SexWare Project.

Elaine Cooper, MB ChB, FFFP

Consultant Advisor in Sexuality Issues, West Hampshire NHS Trust, Hawthorn Lodge, Moorgreen Hospital, Botley Road, West End, Southampton SO30 3JB, UK. Tel: +44 (0) 238047 5157. Fax: +44 (0) 238047 5160. E-mail: dandecooper@yahoo.co.uk

References

1 Disability Discrimination Act 1995, Part 111, Section 21.

2 Sexuality and sexual abuse policy. Tizard Centre, University of Kent, Canterbury, UK, 1995.

3 Good practice in breast and cervical screening for women with learning disabilities. Publication No. 46 and 13. Sheffield: NHSBSP Publications, October 2000. Tel: +44 (0) 114271 1060. E-mail: nhs.screening@sheffield-ha.nhs.uk.www.cancerscreening.nhs.uk

Further reading

1 Cooper E, Guillebaud J. Sexuality and disability - a guide for everyday practice. Abingdon: Radcliffe Medical Press, 1999. 